CASE SERIES

\title{
A Case Series of Children with Developmental Disabilities with Suicidality
}

\author{
Arun Vangili \\ Affiliation: Consultant Psychiatrist - Coimbatore
}

\begin{abstract}
Introduction: Children with developmental disorders are more prone for mental disorders and suicidality. Suicidality often indicates a psychiatric emergency and it is crucial to have an understanding for more effective and efficient intervention. However there is scant literature on the prevalence, risk factors and other characteristics associated with suicidality in this population all over the world and especially in India. Content: In this article we highlight the factors related to suicidality in 3 children with developmental disabilities who were admitted to a tertiary care inpatient psychiatric facility (NIMHANS). Discussion; All three children had multiple psychiatric diagnoses and greater psychosocial adversities. Conclusion: there is a need for reliable screening instruments and routine screening and clinician awareness on this aspect of caring for children with DD
\end{abstract}

\section{INTRODUCTION}

The definition of the term suicidality ranges from occasional thoughts of 'not wanting to live' to serious suicidal attempts. The prevalence of suicidality in youth with Intellectual Disability (ID) and psychiatric comorbidities has been estimated to be between 17 $60 \%{ }^{(1)}$. Suicidal ideation or attempt was found to be 28 times greater in children with Autism Spectrum Disorder (ASD) than in typical children ${ }^{(2)}$. A review article published in 2014 found only 40 publications related to ASD and suicide following a comprehensive search of databases. Most of these publications originated from the west and fewer were from Asian countries ${ }^{(3)}$. In this context, we present a case series of three children with developmental disabilities admitted to in-patient facility for children and adolescents, National Institute of Mental Health and Neurosciences, (NIMHANS), Bangalore.

\section{Case 1:}

L, a 13 yr. old female was brought with significant temper tantrums and difficulties in adjusting at school since the past 5 years. The parents reported that she had difficulty in social interactions, maintaining reciprocal conversation and in understanding nonverbal communication since childhood. She had been refusing to attend school and exhibited verbal and physical aggression when her demands were not met over the last 6 months. She had also been subjected to contact nonpenetrative sexual abuse once at 9 years of age by a stranger.

Corresponding Address:

Dr Arun Vangili

Insight Clinic, Coimbatore

arunvangili@gmail.com
She was diagnosed as having Asperger's syndrome with comorbid ADHD and Depressive Disorder. She harbored suicidal ideations and threatened to kill ${ }^{\mathrm{i}}$ herself whenever she was forced to attend school in the last 6 months. On exploration, she explained that she felt lonely at school as she did not have any friends and that she was having difficulties with academics.

She was started on tablet .escitalopram $5 \mathrm{mg}$ OD and social skills training was begun as part of behavioral management of ADHD. The treating team also liaised with the school to help her obtain services of a resource teacher to help with academics. Discussions about aspects of personal safety were done with the child and her parents. On follow up, child was reported to be going to school for a few hours every day, did not harbor any suicidal ideation and social skills training was ongoing.

\section{Case 2:}

B, a 14 yr. old male was brought with complaints of scholastic difficulties and aggressive behavior towards his parents since 3 years. Parents reported that he had a delay in attaining all developmental milestones, difficulty in processing information and understanding concepts. He was diagnosed to have mild intellectual disability and depressive episode - moderate severity . On detailed assessment it was discovered that his father was not able to come to terms with his limitations and would physically and emotionally abuse him on account of his inability. B would express suicidal ideation frequently as "I will kill you both (parents) and then kill myself as well". On interview he said that he has been ruminating about death more frequently than he expressed. 
The child and family were helped to understand the developmental nature of the problem and individual sessions were held with the father to help him come to terms with the child's impairment. Supportive therapy was done with the child. The family was provided with a perspective of how his strengths could be used and the supports that would help him. At the time of discharge, 8 weeks after admission the B no longer qualified for a diagnosis of depressive episode and did not express any suicidal ideation.

\section{Case 3:}

$\mathrm{R}$, a 12 yr. old male was brought with complaints of repetitive behaviors and hyperactivity. His parents reported extreme restlessness and impulsivity since 5 years of age. He had delay in all developmental milestones and difficulty in understanding concepts. Over the past year, he was defiant and he would do things over and over again despite their instructions. He often expressed thoughts about not wanting to live in the last 1 year. His issues caused significant adjustment problems with peers and he was bullied in school. He was diagnosed to have mild intellectual disability, ADHD and Obsessive Compulsive Disorder (OCD).

He was started on fluoxetine 20mg OD and atomoxetine $18 \mathrm{mg}$ OD for the psychiatric comorbidities. Social skills training was provided and the treating team liaised with the school to ensure implementation of antibullying initiatives at school. On follow up, the child did not have suicidal ideation and was undergoing behavioral therapy for OCD and ADHD.

\section{DISCUSSION}

A Senior Resident and a Consultant Psychiatrist made the diagnosis in each case based on International Classification of Diseases -10. We found a dearth of screening instruments for suicide in developmental disorders. We devised a short screening questionnaire which asked the primary caregivers and the children about 1) any ideas expressed by the child about "death" or "not wanting to live" 2) potentially harmful behaviors suggestive of an attempt like persistent refusal to eat or running towards a busy street saying that he/she wants to be dead 3) any suicidal attempts or self-injurious behavior. This questionnaire is self-developed for easy clinical use and has not been validated. If the parent or child answered in affirmative to any one of these questions then there was further in depth interviewing regarding instances of suicidality

In terms of psychiatric diagnosis, these children had more than one psychiatric disorder and had both externalizing and internalizing disorders in the background of complex psychosocial adversities. It is interesting to note that the caregivers reported externalizing symptoms in all the 3 children. None of these three children had attempted suicide and the parents did not report suicidal ideation spontaneously. It was also not the primary reason for consultation. Similar observations have been reported in a previous study ${ }^{(4)}$.
Suicidal ideations were uncovered only when specific questions were asked during the screening process. This helps us understand that even in these children who present with ii externalizing disorders, it is vital to look for symptoms of internalizing problems and suicidal ideation.

It is well known that parents are usually better informants about symptoms that are tied to external behavior while children are better reporters of internal experiences such as suicidal ideation ${ }^{(5)}$.

Suicide risk assessments in children and adolescents with developmental disabilities pose special challenges due to deficits or delays in language skills, comprehension, poor clinician training and lack of reliable screening instruments ${ }^{(6)}$. Also taboos surrounding talking about suicide and it's association with physical and emotional abuse makes it all the more difficult.Like normally developing children, children with developmental disabilities too exhibit suicidal ideations and actions, hence clinicians should be more aware of this phenomenon.

\section{CONCLUSIONS}

Children with developmental disorders are at risk of psychosocial adversities like abuse and bullying which can lead behavioral and emotional problems. These children are also vulnerable to develop suicidal behaviour and may be unable to express unless specifically iii elicited. Suicide screening should be a part of routine examination for these children, particularly for those who exhibit severe behavioral and emotional problems.

\section{REFERENCES}

1) Ludi, E, Ballard, ED, Greenbaum R, Bridge J, Reynolds W, \& Horowitz, L.Suicide Risk in Youth with Intellectual Disability: The Challenges of Screening. Journal of developmental and behavioral pediatrics 2012; 33(5): 431- 40 .

2) Mayes SD, Gorman AA, Hillwig-Garcia J, \& Syed E. Suicide ideation and attempts in children with autism. Research in Autism Spectrum Disorders 2013; 7(1): 109 - 19.

3) Richa, S., Fahed, M., Khoury, E., \& Mishara, B. Suicide in Autism Spectrum Disorders. Archives of suicide research : official journal of the International Academy for Suicide Research 2014; (July): $37-41$.

4) Koskentausta T, Iivanainen M. \& Almqvist F. CBCL in the assessment of psychopathology in Finnish children with intellectual disability. Research in developmental disabilities 2004;25(4):341-54.

5) Brent D, \& Weersing VR(2008). Depressive Disorders in Childhood and Adolescence. In: Rutter's Child and Adolescent Psychiatry. Fifth Edition, Blackwell Publishing 2008; 587-612.

6) Walters AS, Barrett RP, Knapp LG, \& Borden MC. Suicidal behavior in children and adolescents with mental retardation. Research in developmental disabilities 1995;16 (2) :pp.85-96. 
(C) 2018 IJMHNS

(). 2018 IJMHNS

(C) 2018 IJMHNS 